\title{
Das Entstehen der modernen Medizin - und ein neues Verständnis von Gesundheit und Krankheit: Rudolf Virchow und die Berliner Medizinische Gesellschaft
}

\begin{abstract}
The Emergence of Modern Medicine and a New Understanding of Health and Disease: Rudolf Virchow and the Berlin Medical Society. At the beginning of the 19th century, the most important cities for medicine were Paris and Vienna. Berlin had less than 200,000 inhabitants and no university. Within a short period of time, this changed dramatically - and progress in medicine was a main catalyst. At the end of the 19th century, around two million people lived in Berlin and the city had become the world metropolis of modern medicine. This article examines the main causes and the central figures of this astonishing development. The driving force behind this advance was the rise of a new sort of medicine, i.e., a medicine based on evidence and science. Here, the Berlin Medical Society and its long-time president Rudolf Virchow played a central role. His concept of cellular pathology changed the definitions, methods and understanding of health and disease. Thus, it is no exaggeration to state that Virchow served as one of the most important founding fathers of modern medicine.
\end{abstract}

Zu Beginn des 19. Jahrhunderts waren die Zentren der Medizin Paris und Wien. Berlin spielte kaum eine Rolle. Das änderte sich jedoch schnell. Am Ende des 19. Jahrhunderts war Berlin eine Zweimillionenstadt und Weltmetropole der modernen Medizin. In den dazwischenliegenden hundert Jahren hatte sich die Entwicklung zur neuen wissenschaftsbasierten Medizin vollzogen und Berlin hatte dabei eine führende Rolle gespielt.

Die Universität sowie vielfältige Reformen auch in der Medizin und in den Geisteswissenschaften hat Berlin Napoleon zu verdanken. Nachdem dieser die Preußen 1806 vernichtend geschlagen hatte, wurde der preußische Staat umfassend modernisiert. Nur so, das war den Verantwortlichen klar, konnte man die Oberhoheit der Franzosen wieder abschütteln. Zu den Modernisierungsmaßnah-

Gabriele Laschinski, Berliner Medizinische Gesellschaft

Roman M. Marek, Interdisziplinäre Arbeitsgruppe „Zukunft der Medizin: Gesundheit für alle“, Berlin-Brandenburgische Akademie der Wissenschaften Ivar Roots, Berliner Medizinische Gesellschaft

Ә OpenAccess. (ㄷ 2021 Gabriele Laschinski, Roman M. Marek und Ivar Roots, publiziert von De Gruyter. (cc) BY-NC-SA Dieses Werk ist lizenziert unter einer Creative Commons Namensnennung - Nicht kommerziell - Weitergabe unter gleichen Bedingungen 4.0 International Lizenz. https://doi.org/10.1515/9783110713336-017 
men gehörte auch eine Bildungsreform, für die Wilhelm von Humboldt (17671835) verantwortlich zeichnete. Die Universität wurde von ihm als ein Ort der Forschung und Lehre in Freiheit konzipiert, an dem die Studenten wissenschaftliches Arbeiten und selbständiges Denken lernen sollten. Mit diesem Konzept betrat v. Humboldt damals bildungspolitisches Neuland.

Als die Berliner Universität am 15. Oktober 1810 eröffnet wurde, gehörten der medizinischen Fakultät sechs Ordinarien, ein Extraordinarius und sieben Privatdozenten an. Dekan war der 48-jährige Christoph Wilhelm Hufeland (17621836), königlicher Leibarzt und eine der Schlüsselfiguren des medizinischen Berlins. Gleich im ersten Semester schrieben sich 117 Studenten ein.

Ganz reibungslos verlief die erste Dekade der neuen Einrichtung jedoch nicht. Dafür sorgte zunächst einmal der Mangel an geeigneten Räumlichkeiten, der vor allem die chirurgische Klinik über Jahre dazu zwang, von einer Mietwohnung zur nächsten zu ziehen. Doch auch die Nachricht, es solle ein Lehrstuhl für tierischen Magnetismus eingerichtet und mit Karl Christian Wolfart (1778-1832) besetzt werden, brachte Unruhe in die Fakultät. Diese von Franz Anton Mesmer (17341815) in den 1770er Jahren propagierte Methode hatte zwar schon mancher seiner aufgeklärten Zeitgenossen als Scharlatanerie kritisiert, doch unter dem Einfluss der Naturphilosophie erlebte sie jetzt eine Renaissance. Wolfarts „magnetische Praxis“ gehörte zu den Sehenswürdigkeiten der Stadt. Als Modearzt verkehrte er mit den Vertretern der gebildeten Kreise, mit Heinrich v. Kleist, Joseph v. Eichendorff, Achim v. Arnim, v. Savigny, Schleiermacher. Wolfart wurde schließlich 1817 gegen den Willen der Fakultät Professor.

Schon ein Jahr vorher war mit David Ferdinand Koreff (1783-1851) per Kabinettsorder ein anderer Vertreter der Naturphilosophie der medizinischen Fakultät aufoktroyiert worden. Koreff verkehrte in literarischen Kreisen, war Mitglied des Freundeskreises um E. T. A. Hoffmann, Hausarzt von Wilhelm v. Humboldt und der Vertraute des Staatskanzlers Karl August v. Hardenberg (1750 - 1822), der ihm die Professur verschaffte. Glücklich aber wurde Koreff damit nicht. Vor allem der Anatom Karl Asmund Rudolphi (1771-1832) bot ihm hartnäckig die Stirn: „Unwissenheit heißt die Mutter der mehrsten naturphilosophischen Schriften“ (Rudolphi 1812, S. 41). Als Hardenberg Koreff wegen eines diplomatischen Fauxpas 1822 fallen ließ, ging Koreff nach Paris.

Inzwischen hatte auch die Anziehungskraft des Magnetiseurs Wolfart nach einer undurchsichtigen Affäre um eine Patientin, eine Enkelin von Feldmarschall Blücher, erheblich nachgelassen. Damit hatte die naturphilosophische Fraktion zwei wichtige Exponenten verloren. Die Naturphilosophie geht auf den jungen Friedrich Wilhelm Schelling (1776-1854) zurück und hat in den ersten 30 Jahren des 19. Jahrhunderts die Theorienbildung in der Medizin entscheidend bestimmt. Ihre Anhänger versuchten, die Medizin aus einem generellen Prinzip systematisch 
abzuleiten. Hufeland, als einer ihrer einflussreichsten Vertreter, war Vitalist und vermutete hinter allen Lebensvorgängen das Walten einer allgemeinen Lebenskraft. Er blieb weiter in der Stadt wirksam.

Doch die Naturphilosophie hatte ihre große Zeit hinter sich. Der Philosoph und Naturforscher Henrich Steffens (1773-1845), 1832 auf einen Lehrstuhl der philosophischen Fakultät in Berlin berufen, registrierte: Während vor 20 Jahren begeisterte Mediziner sein Katheder umgaben, waren seine eifrigsten Hörer nun die Theologen.

Der Maaßstab wissenschaftlicher Bestrebungen, der bei einer jeden Beurtheilung in Berlin angelegt wurde, konnte mir nicht günstig sein. [...] Die großen Entdeckungen einerseits in der Physik, dann in der Geologie, endlich in der comparativen Physiologie, hatten einen jeden Keim spekulativer Ansichten erstickt, und die Naturphilosophie ward als ein willkürliches, phantastisches Spiel betrachtet, welches vielleicht hier und da ein dichterisches, keineswegs ein wissenschaftliches Interesse erregen konnte (Steffens 1844, S. 290-291).

\section{Aufbau der wissenschaftsorientierten Medizin}

Dem Ruf nach mehr Wissenschaft und weniger Philosophie und Empirie, der sich mittlerweile unter den jüngeren Medizinern regte, versuchte die Universität bei Neuberufungen Rechnung zu tragen. Mit Johannes Müller (1801-1858) wurde 1833 ein Mann auf den Lehrstuhl für Physiologie und Anatomie berufen, der als Student zwar mit naturphilosophischen Ideen geliebäugelt, sich davon aber inzwischen weitestgehend befreit hatte. Stattdessen vermittelte er in seinen Vorlesungen nun Erkenntnisse der Experimentalphysiologie und exakte Methoden. Müller brachte außerdem seinen Schüler Jacob Henle (1809-1885), einen exzellenten Mikroskopiker, mit. Albert von Kölliker (1817-1905), später Professor für Anatomie und Physiologie, beschreibt in seinen Lebenserinnerungen den prägenden Eindruck dieser neuen Art der Lehre:

Ein Wendepunkt in meinem Leben war Berlin, an welcher Universität ich 3 Semester, vom Herbst 1839 an bis zum Frühling 1841 zubrachte. Hier waren es vor allem Johannes Müller und Jakob Henle, deren Einfluss ein mächtiger war. [...] Ich sehe noch den schmalen langen Vorplatz im Universitätsgebäude neben seinem Hörsaale, in dem Henle in Ermangelung eines anderen Raumes für Demonstrationen, an wenigen, kaum 5 oder 6 Mikroskopen uns die einfachsten, aber in ihrer Neuheit so imponierenden Sachen, Epithelien, Epidermisschüppchen, Flimmerzellen, Blutkörperchen, Eiterzellen, Samentierchen, dann Zupfpräparate von Muskeln, Sehnen, Nerven, Schnitte von Knorpeln, Schliffe von Knochen u.s.w. vorwies und erläuterte, alle Teile selbstverständlich ganz und gar in ihren natürlichen Verhältnissen und ungefärbt. Jetzt wo der jüngste Mediziner schon alles das und viel mehr aus Abbildungen aller Art kennt und die Thatsachen des feinsten Baues des Körpers schon im Gymnasium in aller Munde sind, kann sich nicht leicht einer eine Vorstellung des Ein- 
druckes machen, den damals das erste Erblicken eines Blutstropfens, eines Flimmersaumes, eines Knochenschliffes, einer quergestreiften Muskulatur auf den Studierenden machte und bleibt das Erleben solcher Eindrücke jedem zeitlebens in Erinnerung (Kölliker 1899, S. 8).

Eine weitere wegweisende Neubesetzung war 1840 die Berufung von Johannes Lukas Schönlein (1793-1864) zum Ordinarius und Direktor der Medizinischen Klinik. Er führte die moderne, wissenschaftliche Methode in die Diagnostik ein: Auskultation, Perkussion, Urin- und Blutanalysen, mikroskopische Untersuchungen. Dokumentierte Krankheitsverläufe wurden die Voraussetzung für einen objektiven Befund als Basis für die Diagnose, die ggf. durch eine Obduktion zu kontrollieren ist. Schönlein setzte durch, dass er seine Vorlesung nicht mehr in Latein, sondern auf Deutsch halten durfte - auch dies ein Zeichen dafür, dass in der Medizin klassische Bildung gegenüber naturwissenschaftlicher Methodik an Bedeutung verlor.

Müller und Schönlein werden oft als Begründer der Berliner Schule bezeichnet (z. B. Fischer-Homberger 1977, S. 98). Ihr methodenbasierter Ansatz teilte sich den Studenten als etwas Neues, Zukunftsweisendes mit und versetzte sie in Begeisterung. Der Ruf der Berliner Medizinischen Fakultät verbreitete sich und zog Talente an. Um Müller und Schönlein bildete sich bald ein Kreis hochbegabter Schüler, die hier ihre ersten wissenschaftlichen Erfahrungen sammelten: Rudolf Virchow, Hermann v. Helmholtz, Robert Remak, Emil du Bois-Reymond, Albrecht v. Graefe, Carl Ludwig, Ernst Brücke, Joseph Meyer. Sie und weitere haben die Auffassung von einer, auf physiologischen und pathologischen Grundlagen beruhenden, wissenschaftlichen Medizin in die Welt hinausgetragen und weiter verbreitet. Aus diesem Kreis kam auch eine wichtige Entdeckung, die der Ausgangspunkt für ein epochales Werk wurde: der Müller-Schüler Theodor Schwann $(1810$ - 1882) formulierte 1839 die Theorie, dass Tiere - wie Pflanzen - aus Zellen bestehen.

\section{Die Gesellschaft für wissenschaftliche Medizin und der junge Rudolf Virchow}

Ein Ausdruck der bewussten Positionierung der neuen Medizinergeneration war auch die Gründung der Gesellschaft für wissenschaftliche Medizin im Dezember 1844 durch 18 jüngere Ärzte unter dem Vorsitzenden Friedrich Körte (1818-1914). Die Mitglieder trafen sich alle drei Wochen montags von 7 bis 9 Uhr in einem Vereinslokal zu einer Sitzung, bei der ausschließlich sichere, auf Tatsachen begründete Beobachtungen vorgetragen werden durften. Vage Hypothesen waren verboten. Der Anspruch an die Mitarbeit war hoch: von jedem wurden regelmäßig 
Beiträge erwartet, wer dreimal ohne Entschuldigung fehlte, galt als ausgetreten. Die Vortragenden gehörten zu den besten Männern aus Universität und Praxis, aus diesem Kreis wurden die Mitglieder gewählt.

Bald tauchte ein Name immer häufiger im Programm auf: der des jungen Rudolf Virchow (1821-1902). Virchow hatte 1843 bei Müller promoviert. Er wurde 1846 Prosektor an der Charité und habilitierte sich 1847. Virchow gehörte zu den wenigen genialen Menschen, die eine Sache bis in die Tiefe erfassen, sobald sie diese in Augenschein nehmen, um dann, unbeeindruckt von ihrer Umgebung, voranzuschreiten und neue Wege zu zeigen. Schon der 23-Jährige machte als Festredner bei der Jubiläumsfeier des militärärztlichen Friedrich-Wilhelms-Instituts mit einem Plädoyer für die Medizin als angewandte Naturwissenschaft von sich reden. Während der Märzrevolution 1848/49 gehörte er unter den Medizinern zu den führenden Köpfen. Seine politische Aktivität führte zu Spannungen mit der vorgesetzten Behörde, die Virchow, wenn auch widerstrebend, veranlassten, einen sehr vorteilhaften Ruf nach Würzburg anzunehmen. Allerdings musste er zusichern, sich dort jeglicher agitatorischer Tätigkeit zu enthalten.

So wurde die Zeit in Würzburg für Virchow die wissenschaftlich fruchtbarste seines Lebens. Im Jahr 1856 kehrte er als Professor für Pathologie nach Berlin zurück und wurde mit offenen Armen empfangen. Die Gesellschaft für wissenschaftliche Medizin wählte ihn $1857 \mathrm{zu}$ ihrem Vorsitzenden. Von Februar bis April 1858 stellte er dann in 20 Vorlesungen sein berühmtestes Werk vor: Die CellularPathologie in ihrer Begründung auf physiologische und pathologische Gewebelehre (Virchow 1862).

\section{3 „Omnis cellula e cellula“ - Virchows Verständnis von Gesundheit und Krankheit}

Aufbauend auf der Entdeckung der tierischen Zellen durch Schwann postulierte Virchow, dass die Zelle die Grundeinheit des Lebens sei und jede (pflanzliche, tierische und menschliche) Zelle direkt von einer anderen abstamme. Damit stehen fortan die Zellen im Mittelpunkt seiner forschenden Aufmerksamkeit: Sie sind die Grundelemente des Lebens; ihre dynamischen Wechselwirkungen bestimmen über Krankheit und Gesundheit; sie sind der Ort krankhafter Veränderungen. Krankheit wird damit zu einer Störung der physiologischen Abläufe der Zellen, die mit naturwissenschaftlichen Untersuchungsmethoden beobachtet und analysiert werden kann. Seine epochemachende These „Omnis cellula e cellula“ (,Jede Zelle entsteht aus einer Zelle“) verlegt also den Ursprung des Lebens und der vis vitalis, der Lebenskraft, in die einzelne Zelle. Damit aber formulierte Vir- 
chow einen radikalen Bruch mit religiös und kulturell geprägten Vorstellungen vom Ursprung des Lebens - etwa der bis dato vorherrschenden Ansicht, Zellen entstünden aus einer Art unförmigem Urschleim (dem so genannten Blastem).

Mit seinem Konzept der Cellular-Pathologie legte Rudolf Virchow gleichzeitig die Grundlage für ein neues Verständnis von Gesundheit und Krankheit, das auch in der heutigen biomedizinischen Forschung und Versorgung nichts von seiner Gültigkeit verloren hat: Ohne funktionelle Zellbiologie sind die Teilgebiete Genomik, Proteomik, Metabolomik und das moderne Konzept der Biomarker nicht denkbar (Fischer \& Ganten 2021). Doch während Virchow noch mit dem Mikroskop nach auffälligen oder abweichenden Strukturen auf und zum Teil in Zellen suchen musste, stehen der modernen Zellanalyse ungleich detailliertere und sensiblere Techniken zur Verfügung, um Phänomene auf molekularer oder sogar atomarer Ebene zu erfassen. So erlauben die gegenwärtig verfügbaren Methoden (z. B. Radbruch 2020) Einblicke in die molekularen Strukturen und funktionellen Prozesse von Zellen, die zur Zeit Virchows unvorstellbar waren. Nach wie vor aber gelten Zellen als die Grundeinheiten des Lebens.

Heute lassen sich aus der Analyse einer einzigen Zelle so viele Daten gewinnen, dass diese nur noch mit Hilfe moderner Informationstechnologien und Techniken des maschinellen Lernens interpretiert werden können. Die neuen diagnostischen Möglichkeiten sind heutzutage noch gar nicht zu überblicken. Sicher aber ist, dass die Medizin der Zukunft nicht mehr darauf warten wird, bis ein Mensch mit Schmerzen - d.h. häufig schon zu spät - zum Arzt geht. Neue Technologien wie das Single Cell Sequencing und die daran anschließenden Analysen können Funktionsstörungen oder abweichende Entwicklungen bereits auf zellulärer Ebene aufzeigen, d. h. bevor diese zu klinischen Symptomen führen, die dann ganze Organe betreffen. So eröffnet sich hier eine naturwissenschaftlich fundierte Perspektive auf eine vorbeugende Medizin, die übrigens ganz im Sinne von Virchows Verständnis der „Medizin als sozialer Wissenschaft“ sein dürfte. Denn erst dieses auf Zellbiologie und Biomarkern fußende Verständnis von Krankheit ermöglicht das frühzeitige Erkennen von Krankheitsrisiken und damit langfristig echte Prävention und Global Health.

Bereits zu Virchows Zeiten galt, dass es nicht ausreicht, wenn ein einzelner Kopf eine gute Idee hat. Für das Verwirklichen großer Einfälle und für das Einschätzen der Tragweite neuer Konzepte braucht es meist Zeit. Schon damals und heute vielleicht noch mehr braucht es aber auch Zusammenarbeit und internationale Kooperation - nebst den dafür nötigen Methoden, Strukturen und einer Finanzierung. Virchows Gedankensprung stellt einen Bruch mit bis dato etablierten Vorstellungen dar; für unser heutiges medizinisches Denken ist sein Konzept der Cellular-Pathologie selbstverständlich geworden. Doch für Virchows Zeitgenossen war es eine Erleuchtung, die Ordnung und Zusammenhang in be- 
reits Beobachtetes brachte, wie es der französische Pathologe Victor André Cornil (1837-1908) schildert: „die Zellularpathologie war für uns eine regelrechte Offenbarung. Im Lichte der Zellschäden füllte sich die pathologische Anatomie mit Leben, trat mit der Physiologie in Verbindung und ließ uns die Entwicklung krankhafter Veränderungen verstehen“ (Cornil 1901). Ausgestattet mit einem Empfehlungsschreiben von Claude Bernard machte sich Cornil auf den Weg nach Berlin, um bei Virchow Histopathologie zu erlernen. Er blieb nicht allein, bald strömten Ärzte aus aller Welt zur Ausbildung in Virchows Labor.

Doch war die Pathologie nicht die einzige Attraktion im medizinischen Berlin der 1860er Jahre. Die Chirurgische Universitätsklinik stand seit 1848 unter der Leitung von Bernhard v. Langenbeck (1810 - 1887), einer Leitfigur der deutschen Chirurgie. Seine ausgefeilte, elegante Operationstechnik, noch in einer Ära erworben, in der es keine Narkose gab, zog Schüler aus dem In- und Ausland an. Er hat die bedeutendste deutsche Chirurgenschule hinterlassen. In seiner Privatklinik vor den Toren der Charité wirkte der Augenarzt Albrecht v. Graefe (18281870), auch er ein brillanter Operateur, der die Operationstechnik für Strabismus, Glaukom und Katarakt weitergebracht hat. Von Graefe führte den von Hermann v. Helmholtz entwickelten Augenspiegel in die Praxis ein.

Noch war Berlin nur preußische Hauptstadt, und die Preußen waren bekanntlich sparsam. Selbst einer Weltberühmtheit wie v. Langenbeck gelang es nicht, die zuständigen Stellen von der dringend erforderlichen Modernisierung der Chirurgischen Universitätsklinik zu überzeugen. Diese wurde erst nach der Reichsgründung 1871 in Angriff genommen: Der finanzielle Spielraum war durch die französischen Reparationszahlungen größer geworden, dies kam auch anderen medizinischen Einrichtungen Berlins zugute.

Bald wurde Berlin Zeuge einer neuen Ära: Am 24. März 1882 hielt Robert Koch (1843-1910) vor der Berliner Physiologischen Gesellschaft seinen epochalen Vortrag über den Erreger der Tuberkulose. Koch, seit 1880 am Kaiserlichen Gesundheitsamt, wurde 1885 Direktor des Instituts für Hygiene der Friedrich Wilhelms Universität. Am 4. Oktober 1890 stellte er vor dem 10. Internationalen Medizinischen Kongress in Berlin seine Befunde über die antituberkulöse Wirksamkeit von Tuberkulin vor und löste damit eine beispiellose Pilgerwanderung von Tuberkulosekranken nach Berlin aus. Auch um Koch scharte sich ein Kreis begabter Schüler: Emil v. Behring und Paul Ehrlich, Mitbegründer der modernen Immunologie (Kaufmann 2019), aber auch Friedrich Loeffler, Richard Pfeiffer, August v. Wassermann, Bernhard Nocht, um nur einige zu nennen.

Inzwischen waren seit der Gründung des deutschen Reichs nahezu 30 Jahre vergangen, in denen sich die Einwohnerzahl Berlins mehr als verdoppelt hatte. Dieses explosionsartige Wachstum war hauptsächlich auf Zuzug zurückzuführen, denn als Hauptstadt mit einem großen Dienstleistungssektor und als bedeutender 
Industriestandort übte Berlin eine beträchtliche Anziehungskraft auf auswärtige Arbeitskräfte aus. Wenn stadthygienische Maßnahmen und die medizinische Versorgung der Bevölkerung weitgehend mit dieser rasanten Entwicklung Schritt hielten, war dies zum großen Teil Virchow zu verdanken, der sich um Planung und Ausführung städtischer Krankenhäuser genauso verdient gemacht hat wie u.a. um die Errichtung der Kanalisation. Dafür ernannte die Stadt Berlin ihn $1891 \mathrm{zu}$ ihrem Ehrenbürger.

Rudolf Virchow und Robert Koch sind Jahrhundertgestalten der Wissenschaft; der eine gab der Krankheitslehre mit der Zellularpathologie ein tragfähiges Grundkonzept, der andere erschloss der Medizin mit der Bakteriologie ein neues Gebiet. Beide wirkten über ca. 20 Jahre gemeinsam in Berlin, wenn auch nicht immer einvernehmlich. Sie zogen die Blicke der wissenschaftlichen Welt auf die Stadt; doch sie waren nicht allein. Berlin war inzwischen zum Treffpunkt der Mediziner von Weltrang geworden, Ordinariate und Chefarztpositionen waren heißbegehrt und wurden als Höhepunkt der Laufbahn angesehen (für eine Übersicht vgl. Ebstein 1923). An der Wende zum 20. Jahrhundert leitete Ernst v. Bergmann die chirurgische Klinik der Universität, Ernst v. Leyden und Carl Gerhardt standen den beiden medizinischen Kliniken der Charité vor, Direktor der Gynäkologie war Adolf Gusserow, der Universitätsfrauenklinik Robert v. Olshausen. Otto Heubner war Ordinarius für Kinderheilkunde an der Charité, Wilhelm v. Waldeyer lehrte und forschte in der Anatomie, Max Rubner leitete das Hygienische Institut und Oskar Liebreich, der 1869 mit dem Chloralhydrat das erste moderne Schlafmittel entdeckt hatte, das Pharmakologische Institut. Emil Warburg las Experimentalphysik, Emil Fischer (Nobelpreis für Chemie 1902) organische Experimentalchemie für die Medizinstudenten.

Medizinische Kapazitäten waren jedoch nicht nur in den Universitätsklinika und staatlichen Instituten, sondern auch an städtischen und konfessionellen Kliniken zu finden. Am Augusta-Hospital war der Neurochirurg Fedor Krause tätig, im Krankenhaus der Jüdischen Gemeinde der Chirurg James Israel, im Krankenhaus Friedrichshain leitete Paul Fürbringer die Innere Abteilung, im Krankenhaus Am Urban war Werner Körte Chef der Chirurgie und Prosektor war der berühmte Pathologe Karl Benda. Daneben gab es, vor allem im „Medizinischen Viertel“ rund um die Charité, zahlreiche von bekannten Spezialisten geführte Privatkliniken: die gynäkologischen Kliniken der Gebrüder Landau, von Paul Strassmann und Alfred Dührssen, die dermatologische Klinik von Oscar Lassar und die neurologische von Hermann Oppenheim seien hier beispielhaft genannt.

An all diesen Stätten wirkten, neben Assistenten und Studenten, Gäste aus dem In- und Ausland, die an den Arbeiten teilnahmen und später in ihre Heimat zurückkehrten, um dort im Geist der Berliner Schule weiter tätig zu sein. Das medizinische Berlin war ein hochkompetitives Feld, auf dem sich nur behaupten 
konnte, wer Leistungen und Erfolge vorzuweisen hatte. Der Schauplatz, auf dem dies Kräftemessen stattfand, wo wissenschaftliche Ergebnisse diskutiert, bewertet, gutgeheißen oder verworfen wurden, waren die Sitzungen der Berliner Medizinischen Gesellschaft.

\section{Die Berliner Medizinische Gesellschaft}

Die Berliner Medizinische Gesellschaft war aus der Vereinigung der Gesellschaft für wissenschaftliche Medizin (Vorsitzender Virchow) mit dem 1858 gegründeten Verein Berliner Ärzte (Vorsitzender v. Graefe) hervorgegangen (Artelt 1956). Beim Zusammenschluss im Oktober 1860 hatte die Gesellschaft 204 Mitglieder. Man traf sich anfangs in Vereinslokalen, später im Postfuhramt in der Oranienburger Straße, bis 1892 das von der Deutschen Gesellschaft für Chirurgie neben der chirurgischen Universitätsklinik in der Ziegelstraße erbaute Langenbeckhaus auch der Berliner Medizinischen Gesellschaft eine feste Heimstatt bot. Schon bald wurde dieses Gebäude den beiden Gesellschaften zu klein und sie errichteten in der Luisenstraße gemeinsam das 1915 fertiggestellte Langenbeck-Virchow-Haus.

Den Vorsitz führte bis zu seinem frühen Tod 1870 Albrecht v. Graefe, dann Bernhard v. Langenbeck und ab 1882, bis zu seinem Tode 1902, der gestrenge Virchow. Ihm folgte bis 1907 der Chirurg Ernst v. Bergmann. Die Sitzungen fanden im Winter wöchentlich, im Sommer zweiwöchentlich statt, im Mittel kam man auf 30 Sitzungen im Jahr. Das Auditorium bestand regelmäßig aus mehreren Hundert Zuhörern, denn die Gesellschaft wuchs schnell und hatte 1900 mehr als 1200 Mitglieder. Dazu gehörte nahezu jeder, der im medizinischen Berlin Rang und Namen hatte, die größte Fraktion aber bildeten niedergelassene Ärzte. Man ging in die Sitzungen der Gesellschaft, um das Neueste aus Forschung und Klinik zu hören, denn hier wurden Ergebnisse vorgetragen, die noch nicht publiziert waren. Wer vor diesem Forum sprach, wusste, dass sich Kenner der Materie im Saal befanden. So wurde in wochenlanger Auseinandersetzung um die Bewertung des Behringschen Diphtherieserums und der Kochschen Tuberkulintherapie oder um die Frage nach Indikationsstellung und richtigem Zeitpunkt für eine Appendektomie gerungen. Die Debatte wurde mitstenographiert, und da in Berlin viele der besten medizinischen Köpfe versammelt waren und jeder zu Wort kam, der zu dem Thema etwas beitragen konnte, lesen sich die Sitzungsprotokolle wie gute Übersichtsarbeiten, die Argumente und Gegenargumente sorgsam wiedergeben.

Virchow ragt als der Vorsitzende hervor, der der Berliner Medizinischen Gesellschaft die markanteste Prägung gab. Er wachte über ihre Debatten, sorgte dafür, dass vom Pfad der Wissenschaftlichkeit nicht abgewichen wird und 
Schwachstellen in der Argumentation unerbittlich offengelegt wurden. Geschickt wusste er die Energien der Gesellschaft dahin zu leiten, wo sie am wirksamsten waren.

\section{Virchows Antagonismus zur Bakteriologie}

Bedauerlicherweise verstand sich Virchow nicht gut mit Robert Koch. Die beiden großen Männer hatten seit ihrer ersten Begegnung eine Abneigung gegeneinander, und so kam es, dass Koch 1882 seinen berühmten Vortrag über die Entdeckung des Tuberkelbazillus nicht vor der Berliner Medizinischen Gesellschaft, sondern vor der sehr viel kleineren Berliner Physiologischen Gesellschaft hielt. Möglich, dass der weltberühmte Virchow in dem genialen Koch einen Konkurrenten sah, der den Stellenwert der Bakteriologie überschätzte und mit den Leistungen anderer Disziplinen auch die Virchowsche Zellularpathologie ungerechtfertigt in die zweite Reihe verwies. Virchow stand mit seinen Vorbehalten gegenüber den Bakteriologen auch nicht allein; es hatten sich vielmehr zwei Lager gebildet, zwischen denen es immer wieder zum Schlagabtausch kam.

Bei aller Voreingenommenheit gegen die Bakteriologen erkannte Virchow aber echte medizinische Durchbrüche blitzschnell, wie die heftige Diskussion über die Wirksamkeit des Behringschen Diphtherieserums zeigt.

Nachdem Emil v. Behring (1854-1917) Ende 1890 publiziert hatte, dass die Immunität gegen Diphtherie und Tetanus durch Serum von einem Tier auf das andere übertragen werden kann, lag es nahe, diese Methode auch beim Menschen zu versuchen. Das von Behring selbst entwickelte Serum gegen Diphtherie (Kaufmann 2017) ging im August 1894 bei den Farbwerken Hoechst in Produktion. In Berlin war auch Hans Aronson (1865-1919) bei der Firma Schering die Herstellung eines Diphtherieserums gelungen; es wurde ab März 1894 im Kaiser-undKaiserin-Friedrich-Kinderkrankenhaus erprobt.

Ende November 1894 kam es dann anlässlich eines Referats des Pathologen David Paul Hansemann (1858-1920) vor der Berliner Medizinischen Gesellschaft (Hansemann 1895) zu einer spektakulären Auseinandersetzung. Hansemann behauptete, es fehle der Beweis, dass das Heilserum ein spezifisches Heilmittel gegen Diphtherie sei, eine Haltung, die auch von anderen „Bakteriologie-Skeptikern“ geteilt wurde. Doch diesmal hatten sie Virchow nicht auf ihrer Seite.

Virchow legte bei der Diskussion dieses Vortrages am 5. Dezember 1894 die Diphtherie-Statistik des Kaiser-und-Kaiserin-Friedrich-Kinderkrankenhauses vor. Vom 3. Juni bis 4. August 1894 war das von der Firma Schering kostenlos zur Verfügung gestellte Diphtherieserum angewendet worden; in dieser Periode wurden 72 Kinder geheilt, 10 waren gestorben (Abb. 1, obere Tabelle). Dann starben die 


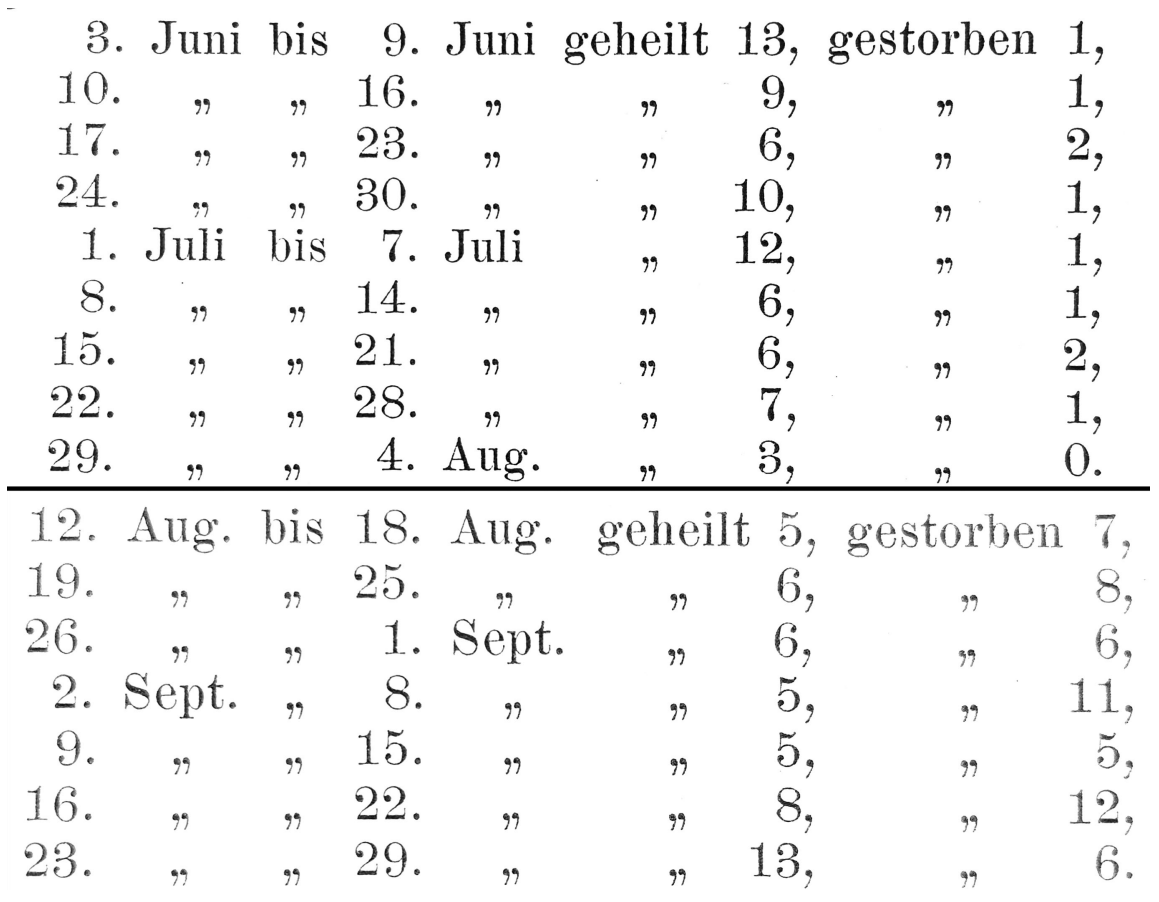

Abb. 1: Sterblichkeit der an Diphtherie erkrankten Kinder im Kaiser-und-Kaiserin-FriedrichKinderkrankenhaus (Direktor: Adolf Baginsky) im Sommer 1894 unter Anwendung des von Emil v. Behring entwickelten Antitoxins. Virchow stellt die Ergebnisse bei der Berliner Medizinischen Gesellschaft vor: „Sie wissen ja, dass in dieser Zeit Herr Dr. Aronson in der Lage war, Heilserum zu liefern, welches er in der Schering'schen Fabrik hergestellt hatte. Dieses Heilserum hatte sich vortrefflich bewährt“ (Berliner medicinische Gesellschaft 1895a, S. 237). (Siehe obere Tabelle)

„Anfang August oder Ende Juli ging das eine der benutzten Pferde plötzlich durch einen Wirbelbruch zu Grunde, ein anderes starb auf andere Weise, - kurz, es war im Augenblick die Quelle für die Gewinnung von neuem Serum in der Schering'schen Fabrik verschlossen. Nunmehr beginnt die andere Periode, wo fast sämmtliche an Diphtherie leidende Kinder, die im Krankenhaus waren, nicht injicirt sind“ (Berliner medicinische Gesellschaft 1895a, S. 237). (Siehe untere Tabelle)

beiden Pferde, die das Serum lieferten, so dass vom 12. August bis zum 29. September kein Serum vorhanden war; ohne Serumgabe wurden 48 Kinder geheilt, 55 starben (Abb. 1, untere Tabelle). Ende September kam Virchow nach Berlin zurück, nahm Einsicht in die Krankenberichte und stellte umgehend Mittel zum Ankauf des Behringschen Serums zur Verfügung. Unter dessen Anwendung wurden vom 14. Oktober bis zum 30. November 110 Kinder geheilt, nur vierzehn verstarben. 
„Alle die theoretischen Betrachtungen, die sich sonst an die blossen Thierexperimente und an die anatomischen Verhältnisse knüpfen, müssen zurücktreten gegenüber diesen, wenn ich so sagen soll, brutalen Zahlen, die so eindringlich sprechen, dass sie meiner Meinung nach alle Widersprüche zurückschlagen“, so Virchow (Berliner medicinische Gesellschaft 1895a, S. 238). Damit hatte sich die führende Autorität der deutschen Medizin, trotz der noch vielen offenen Fragen bezüglich Dosierung, Dauer der Immunität, schädlicher Wirkungen, eindeutig zum Behringschen Diphtherieserum bekannt.

In seinem Schlusswort beklagte Hansemann, dass die Bakteriologen der Diskussion, wie üblich, ferngeblieben wären: „Denn die Erfahrung hat uns doch das gezeigt: alle die wichtigen Fragen, die die Medicin bewegt haben, sind durch unsere Gesellschaft gegangen, sind hier zum Theil bestätigt und auch zum Theil widerlegt worden“ (Berliner medicinische Gesellschaft 1895b, S. 283).

Die Debatte nahm drei Abende in Anspruch. Die Sitzungsprotokolle wurden in der Berliner Klinischen Wochenschrift veröffentlich sowie, jahrgangsweise zusammengefasst, als Verhandlungen der Berliner Medizinischen Gesellschaft in Buchform herausgegeben (Laschinski \& Roots 2018). Sie waren sehr geschätzt, wurden weltweit gelesen und finden sich noch heute in den Archiven der Universitätsbibliotheken rund um den Globus als Zeugnis für die zentrale Stellung, die Berlin in der Medizin für etliche Jahrzehnte innehatte.

Es war deshalb nicht übertrieben, als Virchow 1885 beim 25-jährigen Jubiläum verkündete, dass die Berliner Medizinische Gesellschaft die Stelle einnehme, die anderswo eine Akademie innehabe. Er selbst hat, wie kein anderer Vorsitzender, zu dieser hohen Qualität beigetragen. Als die Welt 1901 seinen 80. Geburtstag feierte, verehrte man in ihm den Mann, der ein halbes Jahrhundert Medizingeschichte maßgeblich geprägt hat (Dhom 2003).

\section{Virchow 2.0: Von der Einzelzellanalyse zum Berlin Cell Hospital}

Doch auch heute noch gilt Rudolf Virchow als der wichtigste Begründer der modernen Medizin.

Dafür gibt es zwei Gründe: Erstens ist das durch ihn geprägte wissenschaftliche und evidenzbasierte Verständnis von Gesundheit und Krankheit bis in die heutige Zeit maßgeblich geblieben für die medizinische Versorgung und die biomedizinische Forschung. Zweitens entspringen seinem Zell-zentrierten Verständnis des Lebens auch heute noch neue Strukturen der Gesundheitsversorgung (Fischer \& Ganten 2021). Der Ausdruck „Virchow 2.0“ verweist darauf, dass 
die von Rudolf Virchow etablierten Grundsätzen der Zelltheorie und Zytologie angesichts der enormen technischen Fortschritte bei der Identifikation, Isolierung, Beobachtung und Analyse einzelner lebender Zellen heute aktueller sind als je zuvor (Helmstetter et al. 2015; Manz et al. 1995; Muñoz et al. 2021; Peine et al. 2013; Radbruch 2020). Neu entwickelte Technologien ermöglichen es heute der biomedizinischen Forschung, mit Hilfe von Einzelzellanalysen den Weg nachzuzeichnen, den einzelne in ihren Funktionen gestörte, d. h. kranke Zellen nehmen, und der schließlich zu systemischen Problemen und letztlich zu einer Krankheit führt, unter der ein Patient leidet. Tatsächlich repräsentiert die Analyse einzelner Zellen mit ihren individuellen Eigenschaften - Einzelzelltechnologien derzeit die medizinische Spitze des wissenschaftlichen Fortschritts (Alliance for Regenerative Medicine 2020; Karaiskos et al. 2017; Pennisi 2018; Walter \& Schickl 2019). Berliner Wissenschaftler waren hier maßgeblich am Aufbau des generellen Human Cell Atlas (Plass et al. 2018) und auch bei spezifischeren Projekten, die einzelnen Organe betreffen wie z. B. der Human Cardiac Cell Atlas, beteiligt. Diese in Berlin gebündelte Expertise führte in Zusammenarbeit mit der Charité -Universitätsmedizin Berlin zu Plänen, ein Berlin Cell Hospital ins Leben zu rufen (Fischer \& Ganten 2021). Hierbei handelt es sich um einen neuen Krankenhaus-Typ, der den jüngsten wissenschaftlichen Stand moderner Zellbiologie für Diagnose und Therapie nutzt und eng an eine Zell-Bank angegliedert ist, die verschiedenste Zelltypen abrufbar hält. Ein wichtiges Ziel wäre hier, spezielle Zellen zur Heilung von Krankheiten zu erzeugen, z. B. für CAR-T-Zell-Therapien (Buchholz et al. 2018; DGHO 2020; Die forschenden Pharma-Unternehmen 2019; Li et al. 2019; Siegmund-Schultze 2019; Singh \& McGuirk 2020; Wörmann 2020). Ein weiterer Meilenstein in diesem Zusammenhang ist LifeTime, eine Initiative, die von mehr als 200 internationalen Wissenschaftlerinnen und Wissenschaftlern initiiert wurde (Rajewsky et al. 2020). Durch interdisziplinäre Zusammenarbeit möchte LifeTime Technologien entwickeln, mit deren Hilfe man einzelne menschliche Zellen während der Entstehung einer Krankheit verfolgen kann. Das Ziel ist, den Einfluss dieser Zellen auf das Krankheitsgeschehen zu verstehen, um dann ihr Verhalten in Richtung auf gesunde oder gar heilende Prozesse umlenken zu können. So wird die einzelne Zelle zum Kristallisationspunkt für die Medizin von morgen - Rudolf Virchow würde sich freuen.

\section{Literatur}

Alliance for Regenerative Medicine (2020): Advancing Gene, Cell, \& Tissue-Based Therapies. ARM Annual Report \& Sector Year in Review: 2019. https://alliancerm.org/sector-report/ 2019-annual-report, besucht am 5.2.2021. 
Artelt, Walter (1956): „Die Berliner Medizinische Fakultät“. In: Ciba-Zeitschrift 78,

S. $2569-2608$.

Berliner medicinische Gesellschaft (1895a): „Discussion über den Vortrag des Herrn

Hansemann vom 28. November: Diphtherie und Diphtherieheilserum“. In: Berliner medicinische Gesellschaft (Hrsg.): Verhandlungen der Berliner medicinischen Gesellschaft aus dem Gesellschaftsjahre 1894, Bd. 25, Teil 1. Berlin: L. Schumacher, S. 232-242.

Berliner medicinische Gesellschaft (1895b): „Discussion über den Vortrag des Herrn Hansemann: Mittheilungen über Diphtherie und das Diphtherieheilserum (Fortsetzung)“. In: Berliner medicinische Gesellschaft (Hrsg.): Verhandlungen der Berliner medicinischen Gesellschaft aus dem Gesellschaftsjahre 1894, Bd. 25, Teil 1. Berlin: L. Schumacher, S. 261- 285.

Buchholz, Christian J./Hartmann, Jessica/Schüßler-Lenz, Martina et al. (2018): „CAR-T-Zell-Therapie: Aussichten und Risiken“. In: Dtsch Arztebl International 115(7), S. 38.

Cornil, Victor André (1901): „Souvenirs d’autrefois“. In: Berliner Klinische Wochenschrift 38, S. 1036.

DGHO (2020): CAR-T-Zelltherapie. Qualitätsgesicherte Durchführung in Deutschland. Stand 5/2020. Berlin: Deutsche Gesellschaft für Hämatologie und Medizinische Onkologie, https://www.dgho.de/publikationen/stellungnahmen/gute-aerztliche-praxis/car-tzelltherapie/car-t-zellen-status-20200722.pdf, besucht am 5.2.2021.

Dhom, Georg (2003): „Rudolf Virchows Spuren in der Medizin nach 100 Jahren“. In: Der Pathologe 24, S. 1-8.

Die forschenden Pharma-Unternehmen (2019): „Die Zukunft der T-Zell-Therapien“. https://www. vfa-bio.de/vb-de/aktuelle-themen/forschung/zukunft-der-t-zell-therapien.html, besucht am 5.2.2021.

Ebstein, Erich (1923): Ärzte-Memoiren aus vier Jahrhunderten. Berlin: Julius Springer.

Fischer, Ernst Peter/Ganten, Detlev (2021): „Die Idee des Humanen“. Rudolf Virchow und Hermann von Helmholtz - Das Erbe der Charité. Stuttgart: S. Hirzel.

Fischer-Homberger, Esther (1977): Geschichte der Medizin (2. Aufl.). Berlin: Springer.

Hansemann, David Paul (1895): „Mittheilungen über Diphtherie und das Diphtherie-Heilserum“. In: Berliner medicinische Gesellschaft (Hrsg.): Verhandlungen der Berliner medicinischen Gesellschaft aus dem Gesellschaftsjahre 1894, Bd. 25, Teil 2. Berlin: L. Schumacher, S. 185-206.

Helmstetter, Caroline/Flossdorf, Michael/Peine, Michael et al. (2015): „Individual T Helper Cells Have a Quantitative Cytokine Memory“. In: Immunity 42(1), S. 108-122.

Karaiskos, Nikos/Wahle, Philipp/Alles, Jonathan et al. (2017): „The Drosophila Embryo at Single-cell Transcriptome Resolution“. In: Science 358(6360), S. 194-199.

Kaufmann, Stefan H. E. (2017): „Emil von Behring: Translational Medicine at the Dawn of Immunology“. In: Nature Reviews Immunology 17(6), S. 341-343.

Kaufmann, Stefan H. E. (2019): „Immunology’s Coming of Age“. In: Frontiers in Immunology 10, S. 1-13.

Kölliker, Albert (1899): Erinnerungen aus meinem Leben. Leipzig: Wilhelm Engelmann. Laschinski, Gabriele/Roots, Ivar (Hrsg.) (2018): Das Entstehen der modernen Medizin. Vorträge vor der Berliner Medizinischen Gesellschaft von 1860 bis 1935. Berlin:

ABW-Wissenschaftsverlag. 
Li, Dan/Li, Xue/Zhou, Wei-Lin et al. (2019): „Genetically Engineered T cells for Cancer Immunotherapy“. In: Signal Transduction and Targeted Therapy 4(1), S. 1-17.

Manz, R./Assenmacher, M./Pfluger, E. et al. (1995): „Analysis and Sorting of Live Cells according to Secreted Molecules, Relocated to a Cell-surface Affinity Matrix“. In: Proc Natl Acad Sci U S A 92(6), S. 1921-1925.

Muñoz, Melba/Hegazy, Ahmed N./Brunner, Tobias M. et al. (2021): „Th2 Cells Lacking T-bet Suppress Naive and Memory T Cell Responses via IL-10“. In: Proceedings of the National Academy of Sciences 118(6), S. e2002787118.

Peine, Michael/Rausch, Sebastian/Helmstetter, Caroline et al. (2013): „Stable T-bet(+) GATA-3(+) Th1/Th2 Hybrid Cells Arise in vivo, Can Develop Directly from Naive Precursors, and Limit Immunopathologic Inflammation“. In: PLoS Biol 11(8), S. e1001633.

Pennisi, Elizabeth (2018): „2018: Breakthrough of the Year. Development Cell by Cell. With a Trio of Techniques, Scientists Are Tracking Embryo Development in Stunning Detail“. Science Magazine. https://vis.sciencemag.org/breakthrough2018/index.html, besucht am 5.2.2021.

Plass, Mireya/Solana, Jordi/Wolf, F. Alexander et al. (2018): „Cell Type Atlas and Lineage Tree of a Whole Complex Animal by Single-cell Transcriptomics“. In: Science 360(6391), S. eaaq1723.

Radbruch, Andreas (2020): „Cell Sorting in Immunological Memory and the Past, Present and Future of Immunology“. https://www.flowcytometry-news.com/category/edito/2020/04/ 07/cell-sorting-in-immunological-memory-and-the-past-present-and-future-ofimmunology-interview-with-prof-dr-andreas-radbruch-scientific-director-germanrheumatism-research-centre-drfz/, besucht am 5.2.2021.

Rajewsky, Nikolaus/Almouzni, Geneviève/Gorski, Stanislaw A. et al. (2020): „LifeTime and Improving European Healthcare through Cell-based Interceptive Medicine“. In: Nature 587(7834), S. 377-386.

Rudolphi, Karl Asmund (1812): „Peter Simon Pallas. Ein biographischer Versuch“. In: Karl Asmund Rudolphi (Hrsg.): Beyträge zur Anthropologie und allgemeinen Naturgeschichte. Berlin: Haude und Spener, S. 1-79.

Siegmund-Schultze, Nicola (2019): „CAR-T-Zellen: Hoffnung und Hype“. In: Dtsch Arztebl International 116(49), S. 2303.

Singh, Anurag K./McGuirk, Joseph P. (2020): „CAR T Cells: Continuation in a Revolution of Immunotherapy“. In: The Lancet Oncology 21(3), S. e168-e178.

Steffens, Henrich (1844): Was ich erlebte. Aus der Erinnerung niedergeschrieben. Bd. 10. Breslau: Josef Mar und Komp.

Virchow, Rudolf (1862): Vorlesungen über Pathologie. Die Cellular-Pathologie in ihrer Begründung auf physiologische und pathologische Gewebelehre. Bd. 1. Berlin: August Hirschwald.

Walter, Jörn/Schickl, Hannah (2019): Einzelzellanalyse in Forschung und Medizin. Eine Stellungnahme der interdisziplinären Arbeitsgruppe Gentechnologiebericht. Berlin: Berlin-Brandenburgische Akademie der Wissenschaften, https://www. gentechnologiebericht.de/fileadmin/user_upload/Webseitendateien/Dokumente/BBAW_ Einzelzellanalyse_A5_PDF-A1-b.pdf, besucht am 5.2.2021.

Wörmann, Bernhard (2020): „Struktur von CAR-T-Zell-Zentren“. 34. Deutscher Krebskongress, Berlin, 19. Februar 2020. https://dkk.conference2web.com/\#!contentsessions/42254, besucht am 5.2.2021. 